\title{
Aportaciones sobre la Medición de Creencias acerca del Maltrato a la Mujer (IBWB) en Población Española
}

\author{
Contributions on the Measurement of Beliefs about the Abuse of Women (IBWB) in \\ the Spanish Population
}

\author{
Andrés Sanchez-Prada ${ }^{1}$, Carmen Delgado-Alvarez ${ }^{2}$, Esperanza Bosch-Fiol ${ }^{3}$ y Victoria A. Ferrer- \\ Perez ${ }^{4}$
}

\begin{abstract}
Resumen
Las creencias y actitudes permisivas hacia la violencia contra las mujeres en la pareja son importantes para su génesis y mantenimiento, por ello es necesario disponer de instrumentos de medida adecuados. El objetivo de este estudio es explorar las fortalezas y debilidades del Inventory of Beliefs about Wife Beating (IBWB), aplicado a 733 estudiantes universitarios/as, junto al Inventario de Pensamientos Distorsionados sobre la Mujer y la Violencia (IPDMV). Los resultados muestran que el modelo con mejor ajuste y teóricamente más coherente para el IBWB conserva 16 ítems, agrupados en tres subescalas (responsabilidad de víctima, maltratador, y política), que correlacionan entre sí y con el resto de variables analizadas de modo teóricamente coherente y coincidente con la literatura científica, y generan diferencias por sexo y conocimiento previo similares a las descritas en dicha literatura. La mayor debilidad de esta nueva propuesta es la relativamente baja consistencia interna de algunas subescalas.
\end{abstract}

Palabras clave: violencia contra las mujeres en la pareja, actitudes, cuestionarios

\begin{abstract}
Permissive beliefs and attitudes towards violence against women in the couple are important for its genesis and maintenance, therefore it is necessary to have suitable measuring instruments. The objective of this study is to explore the strengths and weaknesses of the Inventory of Beliefs about Wife Beating (IBWB), applied to 733 university students, along with the Inventory of Distorted Thoughts on Women and Violence (IPDMV). The results show that the model with the best fit and theoretically most coherent for the IBWB conserves 16 items, grouped into three subscales (victim responsibility, batterer, and policy), which correlate with each other and with the rest of the variables analyzed in a theoretically coherent manner and in line with the scientific literature, and which generate differences by sex and prior knowledge similar to those described in said literature. The greatest weakness of this new proposal is the relatively low internal consistency of some subscales.
\end{abstract}

Keywords: intimate partner violence against women, attitudes, questionnaires

Esta investigación se ha realizado dentro del proyecto investigación FEM2015-63912-P subvencionado por la Agencia Española de Investigación (AEI) y el Fondo Europeo de Desarrollo Regional (FEDER, UE).

\footnotetext{
${ }^{1}$ Facultad de Psicología. Doctor en Psicología. Profesor Encargado de Cátedra. Universidad Pontificia de Salamanca. C/ Compañía, 1-5.37002 Salamanca, España. Tel.: 923277100. Correo: asanchezpr@upsa.es

${ }^{2}$ Facultad de Psicología. Doctora en Psicología. Catedrática. Universidad Pontificia de Salamanca. C/ Compañía, 1-5. 37002 Salamanca, España. Tel.: 923277100. Correo: mcdelgadoal@upsa.es

3 Facultad de Psicología. Doctora en Psicología. Profesora Titular de Universidad. Universidad de las Islas Baleares. Ctra. Valldemossa, km. 7’5. 07122 Palma de Mallorca, España. Tel.971173440. Correo: esperanza.bosch@uib.es

4 Doctora en Psicología. Catedrática. Universidad de las Islas Baleares. Ctra. Valldemossa, km. 7'5. 07122 Palma de Mallorca, España. Tel.: 971173480. Correo: victoria.ferrer@uib.es (Autora de correspondencia)

Revista Iberoamericana de Diagnóstico y Evaluación - e Avaliação Psicológica. RIDEP · No53 · Vol.4 · 49-62 · 2019

ISSN: 1135-3848 print /2183-6051online
} 


\section{Introducción}

Las creencias y actitudes hacia la violencia contra las mujeres en la pareja (VCMP) desempeñan un importante papel tanto en la perpetración de esta violencia como en las respuestas de las mujeres que la padecen, y de la comunidad donde ocurre (Flood \& Pease, 2008, 2009; Wang, 2016). De hecho, el estudio de estas actitudes es considerado esencial para comprender las causas de esta violencia (Fulu, Jewkes, \& García-Moreno, 2013), las respuestas de las mujeres a la victimización que padecen (Sayem, Begum, \& Moneesha, 2012), y también las normas y respuestas sociales hacia esta violencia (Nayak, Byrne, Marti, \& Abraham, 2003), que constituyen el centro de interés de este trabajo. En relación con esto último, cabe recordar que las creencias y actitudes favorables y/o tolerantes hacia la VCMP constituyen uno de los factores de riesgo socioculturales más importantes para su ocurrencia (Dill-Shackleford, Green, Sharrer, Wetterer, \& Shackleford, 2015; Flood \& Pease, 2009; Gracia \& Lila, 2015; Heise \& Katsadam, 2015; Puente, Ubillos, Echeburúa, \& Páez, 2016) $\mathrm{y}$, como tal, tienen un papel relevante en los modelos multicausales explicativos que se barajan actualmente (Bosch \& Ferrer, 2013; Heise, 1998). Así, estos modelos se caracterizan por: incorporar múltiples factores para explicar la génesis y/o mantenimiento de esta violencia; considerar que el género y las relaciones de género desempeñan un papel clave en la violencia ejercida por los hombres hacia las mujeres (APA, 1999); y vincular esta violencia con una sociedad dicotomizada y estructurada desigualmente en función del género (Delgado, 2013), donde las creencias y actitudes sexistas y hostiles hacia las mujeres constituyen, precisamente, la principal justificación para esta asimetría de poder entre hombres y mujeres (Megías \& Montañés, 2010) y pueden generar un clima de tolerancia hacia la violencia contra ellas.

En definitiva, las evidencias disponibles ponen de manifiesto que las creencias y actitudes permisivas hacia la VCMP constituyen elementos clave para su génesis y mantenimiento, lo que justifica ampliamente la necesidad de profundizar en su estudio para avanzar en la comprensión y prevención de este grave problema social.
Consecuentemente, disponer de instrumentos de medida adecuados, fiables y válidos para abordar su estudio científico se convierte en un tema de gran relevancia. En este sentido, cabe recordar que, tradicionalmente, el estudio de las creencias $\mathrm{y}$ actitudes se ha realizado, mayoritariamente, mediante procedimientos directos (también conocidos como medidas explícitas de las actitudes), que indagan sobre las opiniones y valoraciones hacia el objeto de actitud mediante entrevistas, encuestas, cuestionarios o auto informes en los que se solicita a la persona que evalúe ese objeto de actitud (Horcajo, Briñol, Díaz, \& Becerra, 2015).

También las creencias y actitudes sociales o públicas hacia la VCMP han sido estudiadas, básicamente, a través de procedimientos directos como encuestas y cuestionarios (Wang, 2016). Para ello se han diseñado una multitud de instrumentos de evaluación, y, como ocurre en general (López-Cepero, Rodríguez, \& Rodríguez, 2015), muchos de ellos han sido creados ad hoc para determinados proyectos o trabajos en particular, sin que sus características métricas hayan sido siempre suficientemente evaluadas (García-Ael, Recio, \& Silván-Ferrero, 2018; Valor-Segura, Expósito, \& Moya, 2008). Existen, sin embargo, instrumentos ampliamente utilizados y cuyas características psicométricas están adecuadamente contrastadas, como ocurre con el Inventory of Beliefs about Wife Beating (IBWB, Saunders, Lynch, Grayson, \& Linz, 1987). A finales de la década de 1970 y principios de 1980 sus autores/as constataron que, mientras se habían realizado importantes esfuerzos para medir las actitudes hacia la violación (p. ej., Burt, 1980), no existían esfuerzos similares para desarrollar instrumentos que permitieran comprender en toda su complejidad las actitudes sociales generales y específicas hacia la VCMP. De hecho, únicamente pudieron identificar intentos de medir con un único ítem una dimensión de estas actitudes, la aprobación/desaprobación hacia esta violencia (p. ej., Straus, Gelles, \& Steinmetz, 1980), aunque la mayoría de quienes respondían mostraban su desaprobación, por lo que la varianza de tales escalas era, en general, muy baja. Ante esta carencia, Saunders y cols. (1987) desarrollaron el IBWB, una escala cuyo objetivo es medir las múltiples dimensiones de las actitudes y creencias 
hacia las mujeres maltratadas por su cónyuge (casos de "wife beating") en población general. Para ello, construyeron, a partir de ítems de las escalas preexistentes sobre actitudes hacia la violación, de la literatura sobre mujeres maltratadas, y de las consultas a personal que trabajaba con estas mujeres en la clínica y las casas de acogida, 119 ítems que reflejaban diversas dimensiones relativas a este constructo. Posteriormente, realizaron una revisión de estos ítems, y un estudio piloto con alumnado universitario, que les llevó a conservar únicamente 41 ítems, que aplicaron a diferentes muestras (de estudiantes universitarios/as, de población general, de hombres maltratadores, y de profesionales que trabajaban con mujeres maltratadas) para determinar su idoneidad, estructura factorial, validez de constructo, y capacidad para discriminar entre colectivos que habían manifestado previamente actitudes contrapuestas hacia esta violencia. Este proceso desembocó en un cuestionario de 31 ítems: 30 ítems con un formato de respuesta tipo Likert de 7 puntos (desde 1, muy de acuerdo, a 7, muy en desacuerdo), distribuidos en cinco factores o subescalas (Tabla 1), y 1 ítem de respuesta categorial que no se incluye habitualmente en los análisis sobre las características de este instrumento. Tras recodificar los ítems inversos, puntuaciones bajas en las escalas WJ (El maltrato a la mujer está justificado) y WG (Las mujeres obtienen ganancia con el maltrato), y elevadas en las escalas HG (Se debería ayudar a las mujeres maltratadas), OP (El maltratador debe ser castigado) y OR (El maltratador es responsable) indican actitudes menos favorables hacia la VCMP. Estas subescalas muestran, en general, niveles adecuados de validez convergente $y$ discriminante, y una aceptable consistencia interna (habitualmente por encima de .70 para $\mathrm{WJ}$, WG y HG, y algo más baja para OP y OR, como apuntan algunos estudios (p. ej., Berkel, Vandiver, \& Bahner, 2004; Bowen, Gilchrist, \& Beech, 2005). De hecho, muchos trabajos emplean únicamente una o varias subescalas, o versiones abreviadas de este instrumento (Macapagal \& House, 2013). Así, existe una forma corta de 11 ítems (que incluye elementos de las subescalas WJ y HG), y otra de 24 ítems llamada Sympathy for Battered Wives (SBW), que incluye ítems de las escalas WJ, WG y HG (Tabla 1).

Como resumen Macapagal y House (2013), el IBWB ha sido utilizado internacionalmente en estudios con objetivos diversos: establecer diferencias por género (Spencer, Morgan, Bridges, Washburn-Busk, \& Stith, 2017); diferenciar entre varones maltratadores y no maltratadores (Eckhardt, Samper, Suhr, \& Holtzworth-Munroe, 2012); o evaluar cambios tras la participación en programas de intervención para maltratadores (Bowen et al., 2005; Day et al., 2010). También se han desarrollado adaptaciones de este instrumento a distintos idiomas (árabe, portugués, chino, etc.) (Correia, Alves, Morais, \& Ramos, 2015; GarcíaAel et al., 2018; Haj-Yahia, 2003; Haj-Yahia, Sousa, Alnabilsy, \& Elias, 2015; Haj-Yahia \& Shen, 2017; Haj-Yahia \& Zaatut, 2018; Jin, Eagle, \& Yoshioka, 2007; Obeid, Chang, \& Ginges, 2010; Sayem et al., 2012; Zaatut \& Haj-Yahia, 2016), aplicadas, mayoritariamente, en países con culturas fuertemente patriarcales, y que han realizado modificaciones culturales para adaptarlo al entorno (p. ej., Haj-Yahia (2003) elabora una adaptación al árabe que redistribuye los ítems de las escalas WJ y WG, desarrolla una escala para evaluar atribución de responsabilidad a la víctima, y añade nuevos ítems a la escala de atribución de responsabilidad al abusador). En general, estas adaptaciones muestran niveles adecuados de validez convergente (con medidas de hostilidad y violencia contra las mujeres, y actitudes tradicionales hacia los roles de género, por ejemplo), y discriminante (al realizar comparaciones entre población general y/o varones maltratadores $\mathrm{y}$ no maltratadores) $\mathrm{y}$ niveles medio - altos de fiabilidad (entre $.60 \mathrm{y}$ .90, según los casos) (García-Ael, 2018). Por su parte, Craig et al. (2006) adaptaron el IBWB al mainstream actual en VCMP, incorporando el maltrato en casos de parejas no casadas al sustituir términos como esposa (wife) por pareja (partner), o mujer, y golpear (beating) por abusar (abuse), según los casos, y obteniendo en su aplicación en USA niveles de fiabilidad aceptables (.76 para la escala completa, y entre .61 y .77 para las subescalas).

También en España se ha empleado frecuentemente el IBWB para evaluar creencias y actitudes hacia la VCMP con diferentes formatos, 
Tabla 1. Modelos de agrupación de ítems del IBWB en subescalas

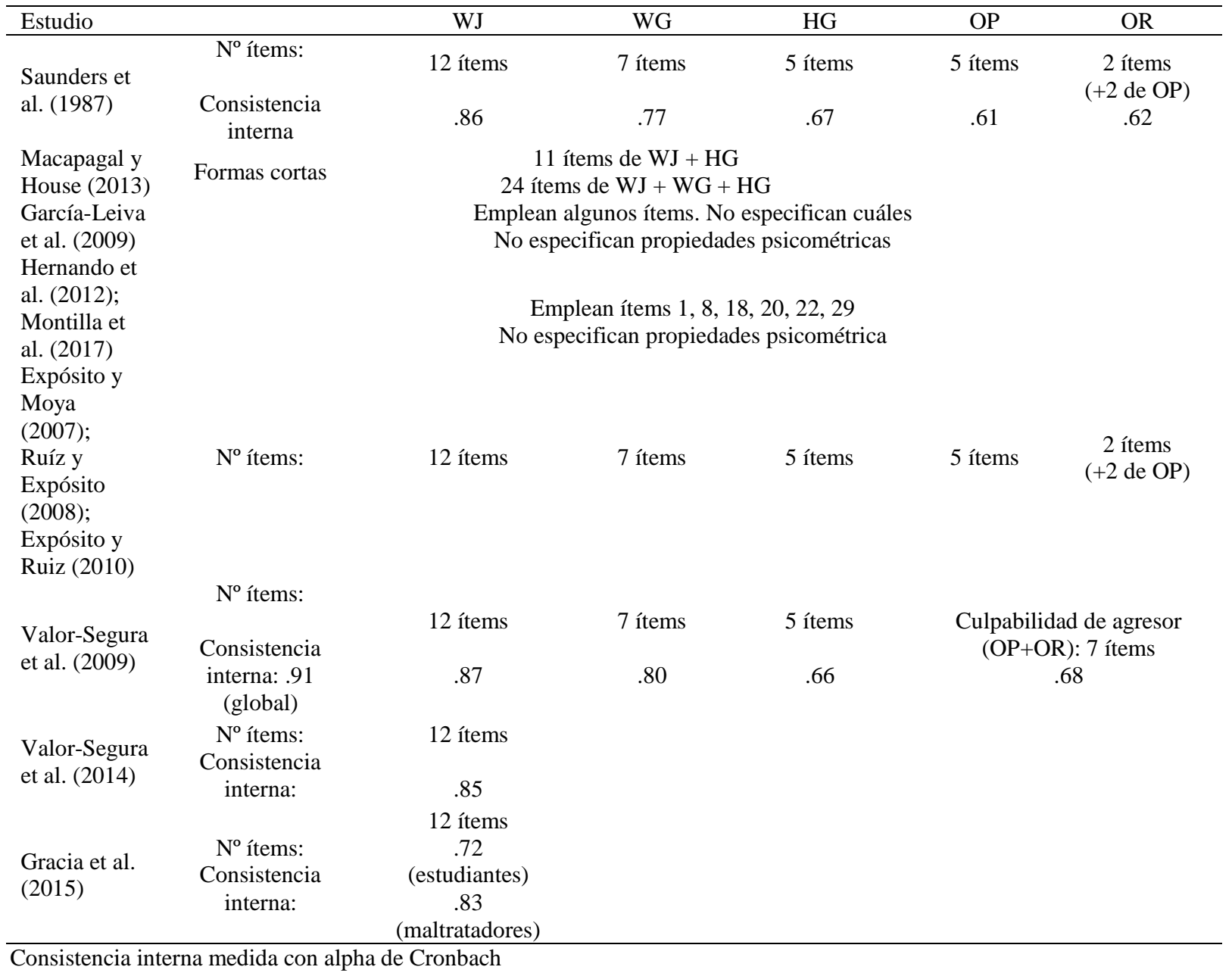

poblaciones y objetivos. Así, por ejemplo, se ha usado el cuestionario completo (con todos sus ítems en su formato original de respuesta) en poblaciones reclusas de hombres y mujeres (Expósito \& Moya, 2007), en hombres maltratadores condenados por delito de maltrato y en situación de suspensión condicional de condena que realizaban un programa de intervención psicosocial con perspectiva de género (Expósito \& Ruiz, 2010; Ruíz \& Expósito, 2008), o en población general adulta (ValorSegura, Expósito, \& Moya, 2009). Otros trabajos han empleado únicamente algunos de sus ítems con estudiantes universitarios (García-Leiva, Palacios, Torrico, \& Navarro, 2009; Hernando, García, \& Montilla, 2012; Montilla, Romero, Martín, \& Pazos, 2017), o una única escala con población general (Valor-Segura, Expósito, Moya, y López, 2014) o con estudiantes universitarios y maltratadores (Gracia, Rodríguez, \& Lila, 2015). En la Tabla 1 se describe la agrupación de los ítems del IBWB en subescalas y la consistencia interna de éstas en las versiones original y abreviada, y en los estudios realizados en España.

En este contexto, partiendo de la consideración de que los/as investigadores/as han de familiarizarse con las medidas disponibles para aquello que quieren medir antes de construir nuevas escalas (Ferrer \& Bosch, 2006), y dada la limitada información psicométrica que aportan las adaptaciones y aplicaciones parciales del IBWB publicadas hasta la fecha, especialmente en lo que respecta a su validez (García-Ael et al., 2018), este estudio tiene como objetivo explorar, a partir de nuevos análisis psicométricos, las fortalezas y debilidades del IBWB en población de habla hispana.

\section{Método}

\section{Participantes}

La muestra fue seleccionada por muestreo no probabilístico incidental entre estudiantes de dos universidades españolas que accedieron a 
participar voluntariamente en la investigación. Participaron un total de 733 sujetos, 267 hombres (36.4\%) y 466 mujeres (63.6\%), con un rango de edad de 18 a 59 años, siendo la media 21.1 años, y la desviación típica 4.05. En cuanto a sus conocimientos sobre VCMP, 454 (61.9\%) habían cursado asignaturas con algún contenido relacionado, y 305 (41.6\%) habían asistido a alguna actividad sobre violencia contra las mujeres: $167(22.8 \%)$ a una, $131(17.9 \%)$ a entre dos y cinco, y siete $(0.9 \%)$ a más de cinco. De los 733 sujetos, $369(50.3 \%)$ tenían pareja, 361 $(49.3 \%)$ no tenían, y tres $(0.4 \%)$ no respondieron a esta pregunta.

\section{Instrumentos}

Para la recogida de información se utilizó:

- Un cuestionario para recopilar datos sociodemográficos de las personas participantes, que incluía, además, una serie de ítems ad hoc:

Dos ítems acerca del grado de conocimiento previo sobre VCMP, a responder en formato dicotómico (Sí/No): uno relativo a formación académica (¿En alguna de las asignaturas que has cursado o estás cursando se incluyen contenidos sobre violencia contra las mujeres?); y otro relativo al ámbito extra-académico (¿Has asistido a alguna actividad (curso, seminario, conferencia...) sobre violencia contra las mujeres?).

Cinco ítems sobre gravedad atribuida a diferentes formas de VCMP (abusos psicológicos, agresiones físicas, abusos sexuales, amenazas de actos de violencia, y restricciones de libertad), a responder en una escala de 1 (nada graves), a 4 (muy graves).

Dos ítems relacionados con opinión sobre la necesidad de implicación del estado en materia de VCMP, uno sobre las leyes para castigar esta violencia, y, sobre la protección y ayuda para mujeres maltratadas, a responder en una escala con cinco categorías de respuesta (muy insuficiente/s, algo insuficiente/s, bastante adecuada/s, muy adecuada/s, excesiva/s, no lo sé).

$$
\text { El Inventario de Pensamientos }
$$

Distorsionados sobre la Mujer y la Violencia (IPDMV, Echeburúa, \& Fernández-Montalvo, 1998), con el formato de respuesta tipo Likert de 4 puntos, de 1 (total desacuerdo), a 4 (total acuerdo), propuesto por Ferrer et al. (2006), donde puntuaciones altas indican aceptación de la violencia y puntuaciones bajas rechazo. Para este estudio se tomaron como referencia las cuatro subescalas correspondientes el modelo revisado de Ferrer, Bosch, Sánchez y Álvarez (2019): inferioridad de la mujer frente al varón (IPDMV1, 7 ítems, $\alpha=.86$ ); culpabilización de las mujeres víctimas de VCMP (IPDMV-2, 7 ítems, $\alpha=.62$ ); violencia como estrategia adecuada para solucionar problemas (IPDMV-3, 5 ítems, $\alpha=.69$ ); y minimización de la violencia contra las mujeres como problema y desculpabilización del maltratador (IPDMV-4, 5 ítems, $\alpha=.53$ ).

- El Inventario de Creencias acerca de del Maltrato a la Mujer (IBWB) de Saunders et al. (1987). Concretamente, se empleó la adaptación de este instrumento al castellano realizada por Expósito y Ruiz (2010), se incluyeron únicamente los 30 ítems con formato de respuesta tipo Likert, y, siguiendo a Craig et al. (2006), se emplearon los términos mujer y pareja (ver apéndice). Para facilitar al máximo la comprensión y las respuestas, se optó por un formato de respuesta tipo Likert de 4 puntos, de 1 (total desacuerdo), a 4 (total acuerdo).

\section{Procedimiento}

Miembros del equipo de investigación solicitaron colaboración al profesorado responsable de diferentes grupos clase. Los/as miembros del equipo de investigación se desplazaban al aula de clase, e informaban a los/as alumnos/as del objetivo de la investigación y de que su participación era completamente voluntaria y no remunerada, y sus respuestas anónimas y confidenciales. Una vez aceptaban participar, los/as alumnos/as firmaban un consentimiento informado, y los/as miembros del equipo investigador les administraban las pruebas descritas en formato lápiz-papel en la misma aula.

\section{Análisis de datos}

Se empleó el análisis factorial confirmatorio (AFC) para valorar el ajuste a los datos del modelo original de cinco factores de Saunders et al. (1987), así como de las diversas variantes, a saber: a) la propuesta de cuatro factores de ValorSegura et al. (2009); b) la forma corta de 24 ítems (Macapagal \& House, 2013; Saunders et al., 1987), considerando tanto un único factor (escala 
SBW) como tres factores (las tres subescalas originales WJ, WG y HG); c) la forma corta de 11 ítems (Macapagal \& House, 2013), considerando también un modelo unifactorial y uno de dos factores (las dos subescalas originales WJ y HG); y la opción unidimensional basada únicamente en la escala WJ (Gracia et al., 2015; Valor-Segura et al., 2014). Se emplearon correlaciones bivariadas para examinar la relación entre las puntuaciones en el IBWB y las variables métricas contempladas en este estudio. Las comparaciones entre grupos según variables no métricas se realizaron mediante MANOVA. Los análisis fueron realizados con SPSS y AMOS 23.

\section{Resultados}

\section{Análisis de la estructura interna del IBWB}

Con el fin de obtener evidencias de validez basadas en la estructura interna (AERA, APA, NCME, 2014) se llevaron a cabo diversos AFCs con los datos obtenidos al administrar el IBWB, usando el método de mínimos cuadrados no ponderados (ULS) para la estimación de los parámetros. Dicho método, basado en la matriz de correlaciones policóricas, está recomendado para variables categóricas, y ha demostrado funcionar adecuadamente con escalas tipo Likert (Forero, Maydeu, \& Gallardo, 2009; Morata-Ramírez, Holgado-Tello Barbero-García, \& Méndez, 2015). Para evaluar el ajuste de los respectivos modelos se emplearon los siguientes criterios $(\mathrm{Hu} \&$ Bentler, 1999; Schermelleh-Engel, Moosbrugger, \& Müller, 2003): como medidas de ajuste absoluto se tomaron el GFI (goodness of fit index $>.90$ ó .95), el AGFI (adjusted goodness of fit index >.90) y el SRMR (standardized root mean square residual <.08 ó .05); y como medida de ajuste incremental se tomó el NFI (normed fit index >.90 ó .95). Se emplearon a su vez dos índices de ajuste parsimonioso: el PGFI (basado en el GFI), y el PNFI (basado en el NFI). En estos dos últimos el umbral de decisión suele ser inferior al habitual .90 , pudiéndose aceptar valores considerablemente menores cuando los modelos a comparar muestran un buen ajuste en otros índices (Muliak et al., 1989).

Como se observa en la Tabla 2, el ajuste estadísticamente más satisfactorio se obtuvo para el modelo de Macapagal y House (2013) de 11 ítems y 2 factores (subescalas originales WJ y HG; con $\alpha=.68$ y $\alpha=.50$, respectivamente). Sin embargo, desde el punto de vista del constructo, ambos factores se focalizan en las mujeres, ya sea como principales responsables o como objetos de protección, invisibilizando a los hombres maltratadores (a los que se refieren las escalas originales OP y OR, ausentes en esta versión). Por ese motivo, se exploraron nuevas combinaciones de ítems que adecuaran el contenido del test al dominio del constructo, sin excluir la responsabilidad del maltratador como dimensión relevante.

Con este objetivo, se utilizó el análisis de componentes principales para alcanzar una combinación de ítems que aglutinase un porcentaje de varianza suficiente tomando un criterio múltiple: análisis paralelo (Horn, 1965), comunalidad y saturaciones, número de ítems por componente, e índice de homogeneidad corregida. Se siguió un proceso iterativo de eliminación de ítems uno a uno hasta obtener una estructura simple y conceptualmente coherente, para lo cual se tomaron como referencia criterios teóricos, además de los estadísticos. Finalmente, se obtuvo una solución de tres componentes y 16 ítems, que explican un $42.43 \%$ de la varianza $(22.11 \%$ el primero; $10.88 \%$ el segundo; y $9.44 \%$ el tercero).

Esta estructura es coherente en cuanto al contenido de los ítems de cada componente: el

Tabla 2. Comparación de modelos de agrupación de ítems del IBWB. Índices de ajuste

\begin{tabular}{|c|c|c|c|c|c|c|c|}
\hline Modelo & $\begin{array}{c}30 \text { ítems } \\
5 \text { factores }\end{array}$ & $\begin{array}{c}28 \text { items } \\
4 \\
\text { factores }^{2}\end{array}$ & $\begin{array}{c}24 \text { ítems } \\
3 \\
\text { factores }^{3}\end{array}$ & $\begin{array}{c}24 \text { ítems } \\
1 \\
\text { factor }^{3}\end{array}$ & $\begin{array}{c}11 \text { ítems } \\
2 \\
\text { factores } 4\end{array}$ & $\begin{array}{c}11 \text { ítems } \\
1 \\
\text { factor }^{4}\end{array}$ & $\begin{array}{c}12 \text { ítems } \\
1 \\
\text { factor }^{5}\end{array}$ \\
\hline GFI & .961 & .960 & .967 & .965 & .986 & .978 & .977 \\
\hline AGFI & .954 & .954 & .961 & .958 & .979 & .968 & .966 \\
\hline PGFI & .813 & .824 & .803 & .810 & .643 & .652 & .676 \\
\hline NFI & .892 & .889 & .924 & .917 & .945 & .913 & .920 \\
\hline PNFI & .806 & .815 & .833 & .838 & .739 & .731 & .753 \\
\hline SRMR & .053 & .053 & .054 & .056 & .050 & .060 & .057 \\
\hline
\end{tabular}




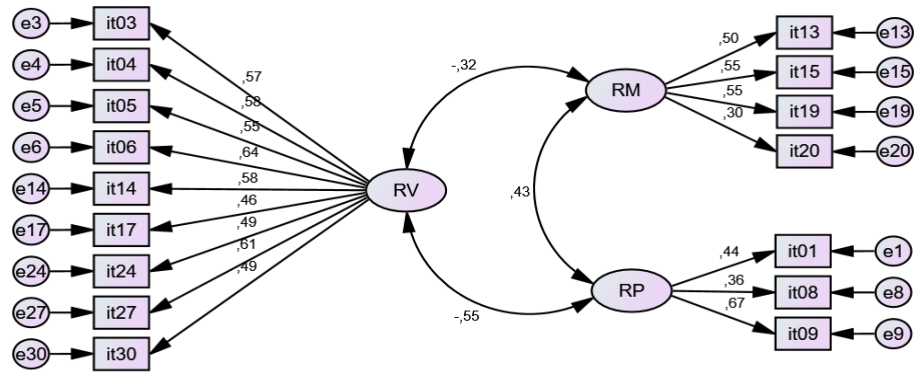

Figura 1. Nuevo modelo para el IBWB (3 factores, 16 ítems)

primero, relativo a la responsabilidad de la víctima (RV, 9 ítems, $\alpha=.79$ ), mantiene cuatro ítems de la subescala WJ del modelo 2factores/11-ítems de Macapagal y House (2013), e incorpora cinco ítems del modelo 5-factores/30ítems de Saunders et al. (1987) (dos de WJ y tres de WG), mejorando considerablemente la consistencia interna; el segundo, relativo a la responsabilidad del maltratador (RM, 4 ítems, $\alpha=.55$ ) incluye todos los ítems de la subescala OR del modelo 5-factores/30-ítems de Saunders et al.; y el tercero, relativo a la responsabilidad política (RP, 3 ítems, $\alpha=.50)$ contiene los mismos ítems que la subescala HG del modelo 2-factores/11ítems de Macapagal y House. Teniendo en cuenta los criterios al uso en investigaciones de carácter básico (Ferrer et al., 2006), los índices de consistencia interna obtenidos, si bien resultan modestos en dos de los tres componentes, pueden ser considerados suficientemente adecuados para el propósito del presente estudio (Nunnally, 1967; Schmitt, 1996). En ese sentido, los índices de homogeneidad corregida resultaron aceptables para todos los ítems, a excepción del ítem 20 $(\mathrm{IHc}=.244)$, que, no obstante, se mantuvo por coherencia teórica, y porque su eliminación afectaba negativamente a la consistencia del componente.

A continuación se sometió a prueba este nuevo modelo mediante AFC por el método ULS, ya descrito, obteniéndose un ajuste satisfactorio (GFI=.980; AGFI=.973; PGFI=.728; NFI=.935; PNFI=.787; SRMR=.048), similar al obtenido con el modelo 2-factores/11-ítems de Macapagal y
House (2013), excepto en los índices de parsimonia, en los que se aprecia un incremento.

En la Figura 1 se muestran los coeficientes estandarizados de regresión y las correlaciones estimadas entre factores. Las cargas factoriales fueron aceptables en todos los ítems, y las correlaciones entre factores concordaron con lo esperable: una correlación positiva entre RM y RP (.43), y correlaciones negativas entre éstas y RV (.32 y -.55 , respectivamente). Por tanto, teniendo en cuenta tanto criterios estadísticos como teóricos, el nuevo modelo presenta ventajas frente al bifactorial de Macapagal y House (2013): mayor adecuación al constructo, mejor consistencia interna del primer factor, y mejores índices de homogeneidad corregida para los ítems más débiles.

\section{Análisis de la correlación con el IPDMV y las variables de gravedad}

Con el fin de obtener evidencias de validez basadas en la relación con otras variables (AERA, APA, NCME, 2014), se obtuvieron las correlaciones de las tres subescalas del modelo propuesto para el IBWB con el IPDMV, como se muestra en la Tabla 3. Se observa, en la línea de lo esperado, que las cuatro subescalas del IPDMV correlacionan positivamente con RV y negativamente con RM y RP, a excepción de la subescala IPDMV4, que no correlaciona con RM. Para esta última se obtienen los índices de correlación más bajos (RM: de -.21 a .03), mientras en el resto de casos se obtienen correlaciones entre moderadas (RP: de -.31 a -.36) $\mathrm{y}$ altas (RV: de .48 a .61). 
Tabla 3. Correlaciones de las subescalas del IBWB con el IPDMV y la gravedad atribuida a la VCMP

\begin{tabular}{|c|c|c|c|c|c|c|c|c|c|}
\hline IBWB & $\begin{array}{c}\text { IPDMV } \\
1\end{array}$ & $\begin{array}{c}\text { IPDMV } \\
2\end{array}$ & $\begin{array}{c}\text { IPDMV } \\
3\end{array}$ & $\begin{array}{c}\text { IPDMV } \\
4\end{array}$ & AP & $\mathrm{AF}$ & AS & AV & RL \\
\hline RV & $.61 * *$ & $.56^{* *}$ & $.48 * *$ & $.26 * *$ & $-.33 * *$ & $-.25 * *$ & $-.24 * *$ & $-.27 * *$ & $-.25 * *$ \\
\hline RM & $-.17 * *$ & $-.21 * *$ & $-.12 * *$ & .03 & .07 & $.17 * *$ & $.09 *$ & $.13 * *$ & $.10 * *$ \\
\hline $\mathrm{RP}$ & $-.36 * *$ & $-.33 * *$ & $-.31 * *$ & $-.09 *$ & $.23 * *$ & $.24 * *$ & $.17 * *$ & $.26 * *$ & $.19 * *$ \\
\hline
\end{tabular}

$* p<.05 ; * * p<.01$.

IBWB-RV: Responsabilidad de víctima; IBWB-RM: Responsabilidad de maltratador; IBWB-RP: Responsabilidad política; IPDMV1: Inferioridad de la mujer; IPDMV2: Culpabilización de mujeres víctimas; IPDMV3: Violencia como estrategia adecuada; IPDMV4: Minimización de VCMP y desculpabilización del maltratador; AP: Abusos psicológicos; AF: Agresiones físicas; AS: Abusos sexuales; AV: Amenazas de actos violentos; RL: Restricciones de libertad.

Tabla 4. Diferencias entre grupos en las subescalas del IBWB

\begin{tabular}{|c|c|c|c|}
\hline VI & Subescala & $\begin{array}{l}\text { Grupos } \\
M(S D)\end{array}$ & Efecto \\
\hline \multirow[t]{6}{*}{ Sexo } & \multirow[t]{2}{*}{ RV } & Hombre: $1.42(.46)$ & $F(1,728)=105.723$ \\
\hline & & Mujer: $1.15(.26)$ & $p=.000 ; \eta^{2}=.127$ \\
\hline & \multirow[t]{2}{*}{$\mathrm{RM}$} & Hombre: 3.45 (.52) & $F(1,728)=.920$ \\
\hline & & Mujer: $3.41(.54)$ & $p=.338 ; \eta^{2}=.001$ \\
\hline & \multirow[t]{2}{*}{$\mathrm{RP}$} & Hombre: $3.54(.52)$ & $F(1,728)=45.893$ \\
\hline & & Mujer: $3.77(.38)$ & $p=.000 ; \eta^{2}=.059$ \\
\hline \multirow{6}{*}{$\begin{array}{l}\text { Conocimiento } \\
\text { previo }\end{array}$} & \multirow[t]{2}{*}{ RV } & Alto: $1.22(.37)$ & $F(1,377)=5.480$ \\
\hline & & Bajo: $1.31(.35)$ & $p=.020 ; \eta^{2}=.014$ \\
\hline & \multirow[t]{2}{*}{$\mathrm{RM}$} & Alto: $3.43(.52)$ & $F(1,377)=.264$ \\
\hline & & Bajo: $3.46(.50)$ & $p=.608 ; \eta^{2}=.001$ \\
\hline & \multirow[t]{2}{*}{$\mathrm{RP}$} & Alto: $3.74(.45)$ & $F(1,377)=5.679$ \\
\hline & & Bajo: $3.62(.48)$ & $p=.018 ; \eta^{2}=.015$ \\
\hline \multirow{6}{*}{$\begin{array}{l}\text { Opinión leyes } \\
\text { anti-violencia }\end{array}$} & \multirow[t]{2}{*}{ RV } & Insuficientes: $1.21(.32)$ & $F(1,338)=12.745$ \\
\hline & & Adecuadas: 1.37 (.48) & $p=.000 ; \eta^{2}=.036$ \\
\hline & \multirow[t]{2}{*}{$\mathrm{RM}$} & Insuficientes: $3.45(.54)$ & $F(1,338)=1.052$ \\
\hline & & Adecuadas: $3.38(.55)$ & $p=.306 ; \eta^{2}=.003$ \\
\hline & \multirow[t]{2}{*}{$\mathrm{RP}$} & Insuficientes: $3.78(.42)$ & $F(1,338)=42.720$ \\
\hline & & Adecuadas: 3.43 (.47) & $p=.000 ; \eta^{2}=.112$ \\
\hline \multirow{6}{*}{$\begin{array}{l}\text { Opinión protección } \\
\text { y ayuda a mujeres }\end{array}$} & \multirow[t]{2}{*}{ RV } & Insuficiente: $1.17(.36)$ & $F(1,303)=8.944$ \\
\hline & & Adecuada: $1.30(.41)$ & $p=.003 ; \eta^{2}=.029$ \\
\hline & \multirow[t]{2}{*}{$\mathrm{RM}$} & Insuficiente: $3.46(.54)$ & $F(1,303)=2.633$ \\
\hline & & Adecuada: $3.36(.57)$ & $p=.106 ; \eta^{2}=.009$ \\
\hline & \multirow[t]{2}{*}{$\mathrm{RP}$} & Insuficiente: $3.84(.39)$ & $F(1,303)=37.881$ \\
\hline & & Adecuada: 3.53 (.46) & $p=.000 ; \eta^{2}=.111$ \\
\hline
\end{tabular}

Por otra parte, y como también se puede observar en la Tabla 3, RV correlaciona negativamente con la gravedad atribuida a la VCMP en sus diferentes formas, relación que se invierte en el caso de RM y RP. Nuevamente, la única excepción se da en RM, que no correlaciona con la gravedad atribuida a los abusos psicológicos. En general, las correlaciones son mayores para RV (de -.24 a -.33), y menores para RM (de .07 a .17).

\section{Análisis de diferencias entre grupos}

Se realizaron diversos MANOVAs, tomando como variables dependientes las puntuaciones en las tres subescalas del IBWB, y como variables independientes los grupos definidos por las siguientes variables no métricas: sexo, conocimiento previo sobre VCMP, opinión sobre las leyes para castigar la VCMP, y opinión sobre la protección y ayuda para mujeres víctimas de VCMP. Puesto que las diferencias entre grupos constituyen una evidencia de validez basada en la relación entre variables (AERA, APA, NCME, 2014), se establecieron dos grupos diferenciados de conocimiento previo: uno de "alto conocimiento" (personas que hubieran cursado alguna asignatura, $y$, además, participado en alguna actividad extra-académica, $n=204$ ), y otro de "bajo conocimiento" (personas que no hubieran cursado ninguna asignatura ni participado en actividades extra-académicas, $\mathrm{n}=175$ ). Igualmente, se crearon dos grupos para cada variable de opinión: quienes consideraban las leyes antiviolencia "muy insuficientes" $(\mathrm{n}=248)$, frente a quienes las consideraban "bastante o muy adecuadas" ( $\mathrm{n}=98)$; y quienes consideraban la protección y ayuda 

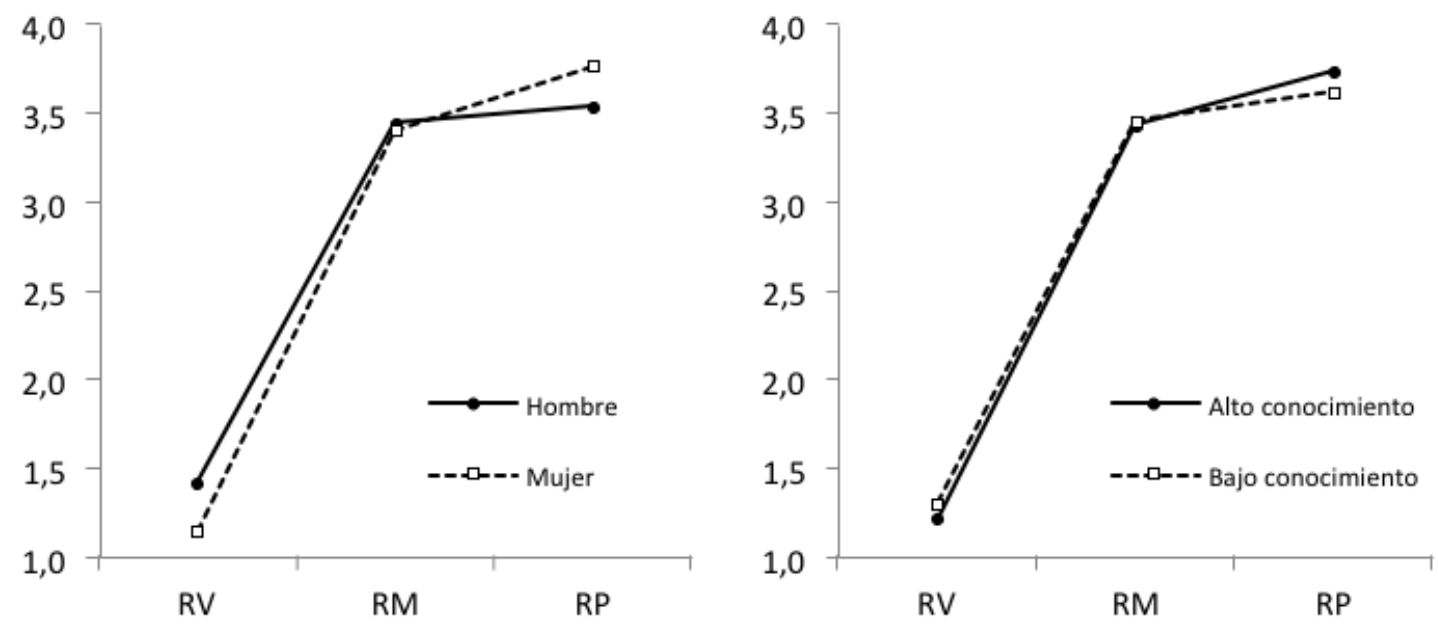

Figura 2. Diferencias según sexo y conocimiento previo
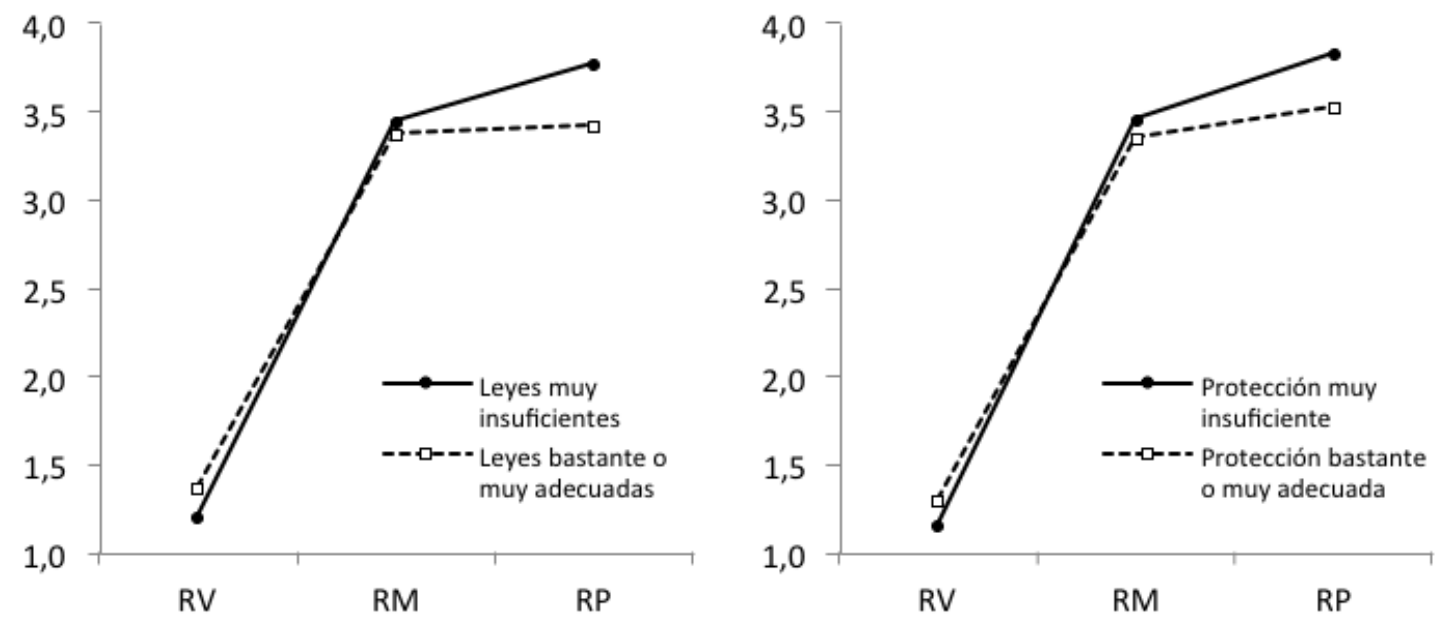

Figura 3. Diferencias según opinión sobre leyes y sobre protección/ayuda

"muy insuficientes" $(\mathrm{n}=144)$, frente a quienes las consideraban "bastante o muy adecuadas" $(n=161)$.

Se empleó la traza de Pillai para los contrastes multivariados, y eta cuadrado parcial para la estimación de los tamaños del efecto. En la Tabla 4 se muestran los efectos intersujetos de cada variable sobre las subescalas del IBWB.

\section{Sexo y conocimiento previo sobre VCMP}

Se halló un efecto significativo del sexo sobre el vector multivariante $(F \quad(3,726)=44.019$; $\left.p=.000 ; \eta^{2}=.154\right)$. Como se muestra en la Tabla 4 , el sexo marcó diferencias significativas en RV y $\mathrm{RP}$, siendo mayor el tamaño del efecto en la primera. Concretamente, los hombres mostraron puntuaciones más altas que las mujeres en $\mathrm{RV}$, y más bajas en RP (Figura 2).
Por su parte, el conocimiento previo sobre VCMP mostró también un efecto significativo, aunque menor $(F \quad(3,375)=3.041 ; \quad p=.029$; $\left.\eta^{2}=.024\right)$, específicamente sobre RV y RP (Tabla 4). En la Figura 2 se muestra cómo el grupo de alto conocimiento obtuvo puntuaciones ligeramente más bajas que el de bajo conocimiento en RV, y más altas en RP.

\section{Legislación y medidas adoptadas por la Administración}

Se halló un efecto multivariante significativo de la opinión sobre las leyes para castigar la $\operatorname{VCMP}\left(F(3,336)=14.650 ; p=.000 ; \eta^{2}=.116\right)$ y de la opinión sobre la protección y ayuda para mujeres víctimas de esta violencia $(F(3,301)=$ 12.759; $\left.p=.000 ; \eta^{2}=.113\right)$. La Figura 3 muestra el sentido de estos efectos. 
Tanto en el caso de la valoración de las leyes anti-violencia como de la protección y ayuda a mujeres, la mayor diferencia entre grupos se dio en RP (Tabla 4), lo cual resulta esperable dada la proximidad conceptual entre variables. Así, quienes valoraron las leyes como "muy insuficientes" mostraron puntuaciones más altas en RP que quienes las consideraron "bastante o muy adecuadas". Se observaron diferencias en el mismo sentido entre quienes consideraron la protección "muy insuficiente" y quienes la consideraron "bastante o muy adecuada".

$\mathrm{Se}$ constataron, asimismo, diferencias significativas en RV para ambas variables independientes. Así, quienes valoraron las leyes como "muy insuficientes" mostraron puntuaciones ligeramente más bajas que quienes las consideraron "bastante o muy adecuadas"; y se observaron diferencias similares entre quienes consideraron la protección "muy insuficiente" y quienes la consideraron "bastante o muy adecuada".

\section{Discusión}

Los resultados obtenidos tras la administración del IBWB muestran que el modelo con mejor ajuste, y que resulta teóricamente más coherente, es aquel que conserva 16 ítems agrupados en tres subescalas: RV, RM y RP. Sobre su coherencia, cabe señalar que la subescala RV se ajusta mejor a la realidad actual de nuestro entorno que las propuestas de Saunders et al. (1987) y Macapagal y House (2013), ya que, más que plantear que la VCMP está justificada (como proponía la escala $\mathrm{WJ}$ original), o que las mujeres obtienen beneficios de la violencia que padecen (como proponía la escala WG), recoge el mito de que las mujeres, de algún modo, son responsables de la VCMP que padecen. La subescala RM constituiría una versión mejorada de las escalas OP (el maltratador debería ser castigado) y OR (el maltratador es responsable) originales, poniendo el acento en la cuestión de la responsabilidad del maltratador. La subescala RP, por su parte, constituiría una versión reducida de la escala HG (las mujeres maltratadas deben ser ayudadas) original, centrada en la necesidad de que el estado se implique en la resolución de este problema social.
Cabe señalar que esta propuesta supera alguna de las limitaciones del IBWB original, apuntadas por autores/as como García-Ael et al. (2018). Así, por ejemplo, al adoptar los términos mujer y pareja, como sugieren Craig et al. (2006), no sólo se evalúan las actitudes hacia la VCMP en el caso de mujeres casadas. Además, las correlaciones entre subescalas concuerdan con la teoría, y con las obtenidas por autores/as como Valor-Segura et al. (2009).

Sin embargo, y en línea con lo ya apuntado por García-Ael et al. (2018), una de las debilidades de este instrumento sigue siendo la relativamente baja consistencia interna de algunas de sus escalas, en nuestro caso de RM y RP. En relación con ello, cabe anotar que nuestra muestra de estudio está compuesta exclusivamente por alumnado universitario, que, en general, presenta un alto rechazo a la VCMP, con puntuaciones bajas en RV y altas en RM y RP, y muy baja variabilidad en sus respuestas. Esto podría ser una limitación, tanto en cuanto a la representatividad de la muestra, como en cuanto a la posibilidad de detectar diferencias y relaciones entre variables, y, junto con su reducido número de ítems, contribuiría a explicar la limitada consistencia interna de las escalas RM y RP, antes mencionada. No obstante, para el propósito actual podemos asumir que "fiabilidades de .60 o .50 resultarán suficientes" (Nunnally, 1967, p. 226).

En cuanto a relación entre las variables medidas con el IBWB y el resto de variables analizadas, las correlaciones con el IPDMV y la gravedad atribuida a la VCMP son coherentes teóricamente, y coinciden con los resultados obtenidos por Saunders et al. (1987) en aspectos como la correlación con las creencias sobre la inferioridad de las mujeres (IPDMV-1), positiva y más elevada con RV, negativa y algo más baja con RP, y aún más baja con RM. Esto último puede estar relacionado con la consistencia interna de las propias escalas RP y, sobre todo RM, a la que ya hemos hecho referencia.

En cuanto al análisis de las diferencias entre grupos, las diferencias obtenidas por sexo son coherentes teóricamente, y también coinciden en gran medida con los resultados obtenidos por Saunders et al. (1987) en cuanto a que los varones obtienen puntuaciones más elevadas en RV (en WJ y WG en el estudio original), y las mujeres en 
RP (en HG en el estudio original), y no hay diferencias entre ellos y ellas en RM (que se daba en el caso de OR en el estudio original). De hecho, en los estudios sobre el tema es habitual observar diferencias en este mismo sentido, esto es, las mujeres tienden a desaprobar la VCMP, y a mostrar actitudes más indulgentes hacia las víctimas, mientras los varones muestran más acuerdo con los mitos hacia esta violencia, y tienden a exonerar a los maltratadores y culpar a las víctimas (Ferrer et al., 2006; Flood y Pease, 2008, 2009; García-Ael et al., 2018; Gracia y Lila, 2015; Valor-Segura et al., 2008; Wang, 2016).

Por lo que se refiere a la comparación según conocimiento previo, los resultados obtenidos son coherentes teóricamente y, en general, concuerdan también con los resultados de estudios previos (Ferrer et al., 2019). Las diferencias halladas entre éstos y otros resultados previos podrían deberse, en el caso de RV, a que anteriormente sólo se tuvo en consideración si las personas habían realizado (o no) alguna actividad relacionada con la VCMP, mientras en este caso se confrontó ausencia de conocimiento previo con alto nivel de conocimiento (operativizado como realizar asignaturas más actividades extra-académicas relativas a VCMP), y en el caso de RM a que las variables medidas no son estrictamente idénticas (responsabilidad del maltratador en el caso del IBWB; desculpabilización del maltratador en el caso del IPDMV).

Finalmente, la comparación en función de la opinión sobre leyes y protección arroja también resultados coherentes teóricamente, de modo que la relación entre esta variable y RP es considerablemente mayor que con las otras dos subescalas, y las personas más críticas con la administración son quienes puntúan más bajo en RV y más alto en RP.

En definitiva, a pesar de las limitaciones señaladas, las principales fortalezas de este estudio son contribuir a suplir la ausencia de información psicométrica sobre el uso IBWB en población de habla hispana, una de las principales dificultades para su uso hasta el momento (García-Ael et al., 2018), y aportar un modelo relevante estadística y teóricamente. Esto nos permite disponer de una adaptación a nuestro entorno de un instrumento como el IBWB, ampliamente contrastado y usado a nivel internacional para el estudio de las creencias y actitudes sociales o públicas hacia la VCMP (Correia et al., 2015; Haj-Yahia et al., 2015, 2017; Spencer et al., 2017). En cualquier caso, cabe remarcar que los resultados obtenidos apuntan la existencia de un amplio margen para la mejora de este instrumento, que podría ir, por ejemplo, en el sentido de: ajustar el lenguaje de los ítems al mainstream actual, más allá de la mera sustitución de términos propuesta por Craig et al. (2006); ampliar el número de ítems de las escalas RM y RP, como proponen García-Ael et al. (2018); o replantear esta última como medida de la atribución de responsabilidades no sólo del estado, sino también de la sociedad en su conjunto, en la línea propuesta por Haj-Yahia (2003), entre otras posibilidades.

\section{Referencias}

AERA, NCME, \& APA (2014). Standards for Educational and Psychological Testing. Washington, DC: AERA

APA (American Psychological Association) (1999). Resolution On Male Violence Against Women. Recuperado de: http://www.apa.org/about/policy/maleviolence.aspx

Berkel, L. A., Vandiver, B. J., \& Bahner, A. D. (2004). Gender role attitudes, religion and spirituality as predictors of domestic violence attitudes in white college students. Journal of College Student Development, 45, 119-133. doi:10.1353/csd.2004.0019

Bosch, E., \& Ferrer, V. A. (2013). Nuevo modelo explicativo para la violencia contra las mujeres en la pareja: el modelo piramidal y el proceso de filtraje. Asparkía, 24, 54-67.

Bowen, E., Gilchrist, E. A., \& Beech, A. R. (2005). An examination of the impact of community-based rehabilitation on the offending behaviour of male domestic violence offenders and the characteristics associated with recidivism. Legal \& Criminological Psychology, 10, 189-209. doi:10.1348/135532505X36778

Burt, M. R. (1980). Cultural myths and support for rape. Journal of Personality and social Psychology, 38(2), 217-230. 
Correia, I., Alves, H., Morais, R., \& Ramos, M. (2015). The legitimation of wife abuse among women: The impact of belief in a just world and gender identification. Personality and Individual Differences, 76, 7-12. doi:10.1016/j.paid.2014.11.041

Craig, M. E., Robyak, J., Torosian, E. J., \& Hummer, J. (2006). A study of male veterans' beliefs toward domestic violence in a batterer intervention program. Journal of Interpersonal Violence, 21(9), 1111-1128. doi:10.1177/0886260506290418

Day, A., Chung, D., O'Leary, P., Justo, D., Moore, S., Carson, E., \& Gerace, A. (2010). Integrated responses to domestic violence: Legally mandated intervention programs for male perpetrators. Trends and Issues in Crime \& Criminal Justice, 404, 1-8.

Delgado, C. (2013). Aspectos cognitivos y emocionales de la violencia de género. En A. Figueruelo, M. del Pozo y M. León (Dirs.), Violencia de género e igualdad. Una cuestión de derechos humanos (pp. 65-79). Granada: Comares.

Dill-Shackeleford, K. E., Green, M. C., Sharrer, E., Wetterer, C., \& Shackleford, L. E. (2015). Setting the stage for social change: Using live theater to dispel myths about intimate partner violence. Journal of Health Communication, 20,969-976. doi:10.1080/10810730.2015.1018622

Echeburúa, E., \& Fernández-Montalvo, J. (1998). Hombres maltratadores. Aspectos teóricos. En E. Echeburúa y P. Corral (Eds.), Manual de violencia familiar (pp. 73-90). Madrid: Siglo XXI.

Eckhardt, C. I., Samper, R., Suhr, L., \& Holtzworth-Munroe, A. (2012). Implicit attitudes toward violence among male perpetrators of intimate partner violence: A preliminary investigation. Journal of Interpersonal Violence, 27(3), 471-491. doi:10.1177/0886260511421677.

Expósito, F., \& Moya, M. C. (2007). Actitudes sexistas y aceptación de la violencia en las relaciones íntimas en una muestra de población reclusa. En R. Arce, F. Fariña, E. Alfaro, C. Civera, \& F. Tortosa (Eds.), Psicología Jurídica, Violencia y Víctimas (pp. 125-132). Valencia: Diputación de Valencia.
Expósito, F., \& Ruíz, S. (2010). Reeducación de Maltratadores: Una experiencia de intervención desde la Perspectiva de Género. Intervención Psicosocial, 19(2), 145-151.

Ferrer, V. A., \& Bosch, E. (2006). Las actitudes sexistas de los maltratadores: El uso de medidas perceptuales como alternativa. Revista Iberoamericana de Diagnóstico y Evaluación - e Avaliação Psicológica, 2(22), 157-181.

Ferrer, V. A., Bosch, E., Ramis, M. C., Torres, E. G., \& Navarro, C. (2006). La violencia contra las mujeres en la pareja: Creencias y actitudes en estudiantes universitarios. Psicothema, 18, 359-366.

Ferrer, V. A., Bosch, E., Sánchez, A., \& Delgado, C. (2019. Beliefs and attitudes about intimate partner violence against women in Spain. Psicothema, 31(1), 38-45. doi:10.7334/psicothema2018.206

Flood, M., \& Pease, B. (2008). Rethinking the significance of attitudes in preventing men's violence against women. Australian Journal of Social Issues, 43, 547-561. doi:10.1002/j.1839-4655.2008.tb00118.x

Flood, M., \& Pease, B. (2009). Factors influencing attitudes to violence against women. Trauma, Violence \& Abuse, 10, 125142. doi:10.1177/1524838009334131

Forero, C.G., Maydeu, A., \& Gallardo, D. (2009). Factor analysis with ordinal indicators: A Monte Carlo study comparing DWLS and ULS estimation. Structural Equation Modeling, 14(4), 625-641. doi:10.1080/10705510903203573

Fulu, E., Jewkes, R., \& Garcia-Moreno, C. (2013). Prevalence of and factors associated with male perpetration of intimate partner violence: Findings from the UN multi-country crosssectional study on men and violence in Asia and the Pacific. Lancet Global Health, 1, 187207. doi:10.1016/S2214-109X(13)70074-3

García-Ael, C., Recio, P., \& Silván-Ferrero, P. (2018). Psychometric properties of the Inventory of Beliefs about Intimate Partner Violence (IBIPV). Anales de Psicología, 34(1), 135-145. doi:10.6018/analesps.34.1.232901

García-Leiva, P., Palacios, M., Torrico, E., \& Navarro, Y. (2009). El sexismo ambivalente: 
¿Un predictor de maltrato? Congreso Latinoamericano de Psicología Jurídica y Forense. Disponible en: http://psicologiajuridica.org/psj210.html

Gracia, E., Rodríguez, C. M., \& Lila, M. (2015). Preliminary evaluation of an analog procedure to assess acceptability of intimate partner violence against women: The Partner Violence Acceptability Movie Task. Frontiers in Psychology, 6, 1567. doi:10.3389/fpsyg.2015.01567

Gracia, E., \& Lila, M. (2015). Attitudes towards violence against women in the EU. Lluxembourg: European Commission Directorate-General for Justice.

Haj-Yahia, M. M. (2003). Beliefs about wife beating among Arab men from Israel: The influence of their patriarchal ideology. Journal of Family Violence, 18(4), 193-206. doi:10.1023/A:1024012229984

Haj-Yahia, M. M., Sousa, C., Alnabilsy, R., \& Elias, H. (2015). The influence of Palestinian physicians' patriarchal ideology and exposure to family violence on their beliefs about wife beating. Journal of Family Violence, 30(3), 263-276. doi:10.1007/s10896-015-9671-4

Haj-Yahia, M. M., \& Shen A. (2017). Beliefs about wife beating among social work students in Taiwan. International Journal of Offender Therapy and Comparative Criminology, 61(9), 1038-1062. doi:10.1177/0306624X15621898

Haj-Yahia, M. M., \& Zaatut, M. A (2018). Beliefs of Palestinian women from Israel about the responsibility and punishment of violent husbands and about helping battered women. Journal of Interpersonal Violence, 33(3), 442467. doi:10.1177/0886260515608802

Heise, L. L. (1998). Violence against women: an integrated ecological framework. Violence Against Women, 4, 262-290.

Heise, L., L., \& Katsadam, A. (2015). Crossnational and multilevel correlates of partnet violence: An analysis of data from populationbased surveys. Lancet Global Health, 3, e332e340. doi:10.1016/S2214-109X(15)00013-3

Hernando, A., García, A. D., \& Montilla, M. V. C. (2012). Exploración de las actitudes y conductas de jóvenes universitarios ante la violencia en las relaciones de pareja. Revista
Complutense de Educación, 23(2), 427-441. doi:10.5209/rev_RCED.2012.v23.n2.40036

Horcajo, J., Briñol, P., Díaz, D., \& Becerra, A. (2015). Actitudes: Concepto, estructura y medición. En J. M. Sabucedo y J. F. Morales (Coords.), Psicología social (pp. 117-136). Madrid: Ed. Médica Panamericana.

Horn, J. L. (1965). A rationale and test for the number of factors in factor analysis. Psychometrika, 30, 179-185. doi:10.1007/BF02289447

Hu, L., \& Bentler, P.M. (1999). Cut-off criteria for fit indexes in covariance structure analysis: Conventional criteria versus new alternatives. Structural Equation Modeling, 6(1), 1-55. doi:10.1080/10705519909540118

Jin, X., Eagle, M., \& Yoshioka, M. (2007). Early exposure to violence in the family of origin and positive attitudes toward marital violence: Chinese immigrant male batterers vs. controls. Journal of Family Violence. 22, 211-222. doi:10.1007/s10896-007-9073-3

López-Cepero, J., Rodríguez, L., \& Rodríguez, F. J. (2015). Evaluación de la violencia de pareja. Una revisión de instrumentos de evaluación conductual. Revista Iberoamericana de Diagnóstico y Evaluación - e Avaliação Psicológica, 2(40), 37-50.

Macapagal, K., \& House, A. (2013). Critical synthesis package: Inventory of beliefs about wife beating (IBWB). MedEdPORTAL, 9, 9610. doi:10.15766/mep_2374-8265.9610

Megías, J. L., \& Montañés, P. (2010). Prevención primaria de la violencia de género. En $\mathrm{S}$. de Lemus y E. Ryan (Eds.), Coeducación. Propuestas para alcanzar la igualdad de género desde las aulas (pp. 127-163). Granada: Universidad de Granada.

Montilla, M. V. C., Romero, C., Martín A., \& Pazos, M. (2017). Actitudes de los adolescentes acerca de la violencia en parejas de jóvenes. Revista de Orientación Educacional, 31(59), 53-72.

Morata-Ramírez, M. A., Holgado-Tello, F. P., Barbero-García, I., \& Méndez, G. (2015). Análisis factorial confirmatorio. Recomendaciones sobre mínimos cuadrados no ponderados en función del error Tipo I, de Ji cuadrado y RMSEA. Acción Psicológica, 12(1), 79-90. doi:10.5944/ap.12.1.14362 
Muliak, S. A., James, L. R., Van Alstine, J., Bennett, N., Lind, S., \& Stilwell, C. D. (1989). Evaluation of goodness-of-fit indices for structural equation models. Psychological Bulletin, 5(3), 430-455.

Nayak, M. B., Byrne, C. A., Martín, M. K., \& Abraham, A. G. (2003). Attitudes toward violence against women: a cross-nation study. Sex Roles, 49, 333-342. doi:10.1023/A:1025108103617

Nunnally, J. C. (1967). Psychometric Theory. New York: McGraw-Hill.

Obeid, N., Chang, D. F., \& Ginges, J. (2010). Beliefs about wife beating: An exploratory study with Lebanese students. Violence Against Women, 16, 691-712. doi:10.1177/1077801210370465

Puente, A., Ubillos, S., Echeburúa, E., \& Páez, D. (2016). Risk factors associated with the violence against women in couples: A review of meta-analysis and recent studies. Anales de Psicología, 32(1), 295-306. doi:10.6018/analesps.32.1.189161

Ruíz, S., \& Expósito, F. (2008). Intervención con hombres en suspensión condicional de condena por violencia de género. Anuario de Psicología Jurídica, 18, 81-89.

Saunders, D. G., Lynch, A. B., Grayson, M., \& Linz, D. (1987). The inventory of beliefs about wife beating: The construction and initial validation of a measure of beliefs and attitudes. Violence \& Victims, 2(1), 39-57.

Sayem, A. M., Begum, H. A., \& Moneesha, S. S. (2012). Attitudes towards justifying intimate partner violence among married women in Bangladesh. Journal of Biosocial Science, 44(6), 641-660. doi:10.1017/s0021932012000223

Schermelleh-Engel, K., Moosbrugger, H., \& Müller, H. (2003). Evaluating the fit of structural equation models: Tests of significance and descriptive goodness-of-fit measures. Methods of Psychological Research Online, 8(2), 23-74.

Schmitt, N. (1996). Uses and abuses of Coefficient Alpha. Psychological Assessment, 8(4), 350-353. doi:10.1037/1040-3590.8.4.350

Spencer, C. M., Morgan, P., Bridges, J., Washburn-Busk, M., \& Stith, S. M. (2017). The relationship between approval of violence and intimate partner violence in college students. Journal of Interpersonal Violence. Published first on line. doi:10.1177/0886260517731315

Straus, M. A., Gelles, R. J., \& Steinmetz, S. K. (1980). Behind the closed doors: Violence in the American family. New York: Doubleday.

Valor-Segura, I., Expósito, F., Moya, M., \& López, K. (2014). Violencia hacia la mujer en España y Cuba: Una misma realidad, dos visiones diferentes. Revista de Psicología Social, 29(1), 150-179. doi:10.1080/02134748.2013.878573

Valor-Segura, I., Expósito, F., \& Moya, M. (2008). Atribución del comportamiento del agresor y consejo a la víctima en un caso de violencia doméstica. Revista de Psicología Social, 23(2), 171-180. doi:10.1174/021347408784135896

Valor-Segura, I., Expósito, F., \& Moya, M. (2009). La dependencia específica en la pareja y la tolerancia hacia el abuso en las relaciones íntimas. En F. Expósito y S. de la Peña (Eds.), Psicología Jurídica de la violencia y la delincuencia: Actuaciones con víctimas $y$ victimarios (pp. 47-54). Murcia: Universidad de Murcia.

Wang, L. (2016). Factors influencing attitude toward intimate partner violence. Aggression \& Violent Behavior, 29, 72-78. doi:10.1016/j.avb.2016.06.005

Zaatut, A., \& Haj-Yahia, M. M. (2016). Beliefs about wife beating among Palestinian women from Israel: The effect of their endorsement of patriarchal ideology. Feminism \& Psychology, 26(4), 405-425. doi:10.1177/0959353516647071 